\title{
The Lung Immune Niche in Tuberculosis: Insights from Studies on Human Alveolar Macrophages
}

\author{
Soumya Chatterjee ${ }^{1}$
}

Published online: 22 April 2015

(C) Springer International Publishing AG (outside the USA) 2015

\begin{abstract}
Tuberculosis (TB) is a disease that continues to cause global mortality and morbidity. Transmission of $\mathrm{Myco-}$ bacterium tuberculosis $(\mathrm{Mtb})$ occurs by aerosol transmission from an infected case, making the lung the primary portal of entry for the bacterium. Alveolar macrophages are the frontline cells involved in the control of subsequent replication and spread of disease. Although animal models have provided important information in the field of macrophage immunology and cell biology, human TB disease has several unique features. Therefore, an understanding of human alveolar macrophage biology and their interactions with Mtb is important, not only to understand how to address TB control at various stages of the disease but also to develop optimal future vaccination and drug strategies. Despite limitations of human sample access, technical expertise in cell processing, and access to reagents, significant advances have been made in the field providing critical insights into host-pathogen interactions This review describes the unique features and the immune responses of human alveolar macrophages in the context TB infection.
\end{abstract}

Keywords Tuberculosis immunology $\cdot$ Human alveolar macrophages $\cdot$ Macrophage phenotype $\cdot$ Macrophage immunity

This article is part of the Topical Collection on Bacterial Tropical Medicine

iThenticate: $10 \%$

Soumya Chatterjee

chatterjees3@mail.nih.gov

1 Laboratory of Parasitic Diseases, National Institute of Allergy and Infectious Diseases, National Institutes of Health, 4 Center Drive, Room B1-09, NIH, Bethesda, MD 20892, USA

\section{Introduction}

Tuberculosis (TB) caused by Mycobacterium tuberculosis (Mtb) remains one of the most important causes of global morbidity and mortality, affecting nine million people in 2013 and causing 1.5 million deaths [1]. The bacterium is spread by the aerosol route from an infected individual, and the lungs are the primary portals of entry. Alveolar macrophages (AM) are the primary lung resident phagocyte/ antigen-presenting cells that are involved in the initial immune response to the bacterium, and the Mtb-AM interaction is critical to determine the subsequent fate of infection, i.e., infection clearance vs. disease progression. Although experimental animal models of TB have provided important insights into how AMs interact with Mtb, the exact molecular mechanisms involved in these interactions continue to be defined. Additionally, human AMs appear to possess unique characteristics compared to in vitro-generated monocyte-derived macrophages (MDMs) or mouse macrophages suggesting that human AMs might be better adapted for clearance of small particles $(<5 \mu \mathrm{m})$ and limit uncontrolled inflammation [2]. This widely held view has recently been questioned by studies that AMs might have a proinflammatory phenotype at baseline [3] Human AM studies, therefore, provide important information about the processes involved that may benefit either the bacterium or the host. Access to AMs obtained by bronchoalveolar lavage is difficult, however, especially with resource and personnel constraints in TB-endemic areas. These in vitro assessments also do not take into account the immune milieu of the alveolar space in the lung where these cells reside and function. There is renewed appreciation of how factors like alveolar epithelial cells and surfactant, as well exposure to environmental pollutants and smoke can alter immunological responses in these cells $[4,5]$. This review focuses on studies performed specifically on the immunological interactions of 
human alveolar macrophages with Mtb and attempts to identify future directions of research in the field.

\section{Phenotypic and Functional Characteristics of Human AMs}

Approximately $90-95 \%$ of the cells obtained by research bronchoscopy and bronchoalveolar lavage (BAL) are AMs in normal individuals [6-8]. However, in patients with active pulmonary tuberculosis who underwent bronchoscopy and subsequent assessment of the cellular fraction by cytospin, AMs comprised about $67 \%$ of the total cellular fraction with about $16 \%$ lymphocytes and $3 \%$ neutrophils [9]. Peripheral blood monocytes migrating and differentiating into the airways are thought to be the major precursors for AMs [10]. It has also been demonstrated, however, that mononuclear phagocytes present in the lung can divide in the alveolus in response to local inflammation [11]. There have been a number of different studies that have evaluated the phenotypic characteristics of the macrophages obtained by BAL, and different sets of markers have been utilized to define these cells [12-15]. It is well established from most studies that in healthy individuals, the expression of multiple surface markers in AMs are different from blood monocytes [12]. Most studies show that these cells are $\mathrm{FSC}^{\text {hi }}$ $\mathrm{CD} 14^{\mathrm{lo}} \mathrm{CD} 15^{\mathrm{lo}} \mathrm{CD} 16^{\mathrm{hi}} \mathrm{CD} 44^{\mathrm{hi}} \mathrm{HLA}-\mathrm{DR}+\mathrm{CD} 206+$. Additionally, they also seem to have increased expression of CD80, CD86, and CD40 and lower expression of CD11a and $\mathrm{CD} 11 \mathrm{~b}$. Although decreased mRNA expression of TLR2, 4, and 9 was noted in AMs compared to monocytes, increased surface expression of TLR9 was noted in AMs [16]. Recent flow cytometric studies in the context of HIV have identified a population of macrophages (termed small macrophages) that express macrophage-specific phenotypic markers CD71 and CD206 and CD16. The intensity of expression of CD206 was, however, significantly decreased compared to the predominant population of large macrophages [17]. The authors hypothesized that these small macrophages may be the previously described immature macrophages with these small macrophages demonstrating reduced phagocytic capacity (as measured by phagosomal bulk proteolytic activity) compared to the large macrophage population in HIV-uninfected subjects [18]. Recently, transcriptional profiling showed that genes in human AMs compared to MDMs were significantly enriched for immune, inflammatory, and defense responses and cytokine activity [19]. This included enrichment of the NF-kB family members. Interestingly, basal proinflammatory gene expression in these cells was decreased after a 48-h culture with increased responsiveness to stimulation by LPS or poly I:C. In a separate study, human AMs showed upregulation of MHC class II genes [3]. Additional genes of diverse function distinguished AM from MDM, including MARCO, a bacteria-binding receptor expressed in certain subsets of MDM [20] and CCL18. These findings are especially important as it appears that macrophage plasticity and heterogeneity depend on the inflammatory milieu in the lung, contradicting the generally held belief that AMs might be alternatively activated at baseline [21-24]. Additionally, the lung microenvironment appears to play an important immunomodulatory role in shaping immune responses by AMs. Surfactant-associated pulmonary collectins surfactant protein A (SP-A) and surfactant protein D (SP-D) regulate the early interaction of the bacilli with resident phagocytes $[25,26]$. SP-A appears to downmodulate AM responses to Mtb by decreasing generation reactive nitrogen intermediates (RNIs) and reactive oxygen intermediates (ROIs) in response to stimuli [25]. SP-D appears to decrease phagocytosis of Mtb in a mannose receptor (CD206) dependent manner by binding to the mannose caps of the Mtb cell wall mannosylated lipoarabinomannan (ManLAM) [27]. Opsonization of Mtb by SP-D leads to increased phagosome-lysosome fusion and decreased bacterial survival.

Alveolar macrophages, therefore, are unique from blood monocytes and seem to have an increased surface expression of adhesion markers (CD44) and receptors for particle and pathogen uptake (i.e., CD206). AM heterogeneity and plasticity in response to different pro- or anti-inflammatory stimuli (especially surfactant-derived proteins) are also an evolving field of research, and further studies are needed to better understand how these cells control mycobacterial replication under differing immune-activating conditions.

\section{Uptake of Mycobacteria}

Human AMs have been thought of as efficient phagocytes given their location in an environment where there is constant exposure of these cells to foreign matter including microbes. Phagocytosis by these cells can proceed in an opsoninindependent fashion [28]. It has been demonstrated that these cells are more efficient than human monocytes at Mtb uptake and that, in addition to the heightened expression of surfactant protein A receptor A [29], and mannose receptor [30], complement receptor 4 and TNF- $\alpha$ might be involved in these cells being more efficient phagocytes and limiting Mtb growth and replication [31]. In pulmonary TB patients, dendritic cellspecific intercellular adhesion molecule-3 grabbing nonintegrin (DC-SIGN) appears to be overexpressed in macrophages, and anti-DC-SIGN antibodies were able to significantly inhibit Mtb binding in the DC-SIGN-expressing AMs [32]. Thus, AMs appear to be efficient at mycobacterial uptake, but the eventual outcome of infection is determined by 
their interaction with the phagolysosome which is discussed below.

\section{Cytokine and Chemokine Production}

AMs are capable of producing cytokines, like IL-12 and additional cyto, and chemokines such as TNF- $\alpha$, IL-1, IL-6, IL15 , and IL-18 in response to Mtb [33-36]. Although the importance of the IL-12-IFN- $\gamma$-TNF- $\alpha$ axis in Mtb control is well established from human and animal studies [37, 38], increased levels of TNF have been found with increasing multiplicity of infection (MOI) of live Mtb in AMs and was found to positively correlate with growth of virulent Mtb (H37Rv) suggesting that TNF may contribute to immunopathology by directly supporting bacterial growth [39]. Furthermore, in studies of healthy contacts of subjects with active TB, CD4+ $\mathrm{T}$ cell, AM co-cultures lead to increased IFN- $\gamma$ production, improved control of bacterial growth was only seen in CD8:AM co-cultures in these subjects [40]. AMs appear to release higher levels of monocyte chemotactic protein-1 (MCP-1) and macrophage inflammatory protein-1alpha (MIP-1alpha) compared to blood monocytes and also express higher levels of regulated on activation normal $\mathrm{T}$ cell expressed and secreted (RANTES) in response to both virulent and avirulent Mtb strains [41]. Additionally, when gene expression in human AMs was compared in response to virulent Mtb strain H37Rv vs. H37Ra, it was observed that IL-23 p19 subunit was the most highly upregulated by infection with H37Rv, followed by genes for GM-CSF, the neutrophil chemotactic molecule CXCL1 (GRO $\alpha$ ), IL-6, and TNF- $\alpha$ [42]. Detectable amounts of IL-23 but not IL-12 were found in both human AMs and monocyte cultures in response to mycobacteria in this study, suggesting an important role of this cytokine in the initial responses against Mtb. In in vitro studies, therefore, AMs appear to be fully capable of mounting a pro-inflammatory response which is modulated by the virulence of the mycobacterial strain studied. In vitro studies also suggest that they have an important role in chemotaxis of $\mathrm{T}$ cells, monocytes, and neutrophils.

\section{Phagolysosome and Autophagy}

Intracellular pathogen elimination is dependent on the gradual acidification of the phagosome. Mtb is a highly successful pathogen that is able to manipulate and prevent phagosome acidification and maturation of this compartment [43]. This phenomenon was recently demonstrated to be controlled by Abl tyrosine kinases and could be reversed by the Abl tyrosine kinase inhibitor imatinib [44]. Mtb can also impair phagolysosomal fusion. This phenomenon has been demonstrated for human AMs as well [45] — a phenomenon called lysosomal spreading. It is now appreciated that stimulation of autophagy might be important for phagolysosome maturation and elimination of bacteria. Activation of nucleotide-binding oligomerization domain 2 (NOD2) receptor, which has been shown to be expressed in AMs [46] and senses peptidoglycan, appears to be important in controlling Mtb replication in these cells by induction of autophagy [47]. Similar induction of autophagy and Mtb growth control has been demonstrated by activation of NOD1 [48]. Regulatory cytokines like IL10 have, on the other hand, been shown to inhibit phagosomal maturation [49]. Acidification of the phagolysosome and induction of pathways of autophagy are therefore potentially two important areas which can be targeted by novel therapeutics aiming to manipulate the immune system for optimal TB control.

\section{Apoptosis}

Apoptosis of macrophages infected with Mtb has been thought of as a phenomenon beneficial to the host by preventing a cellular environment conducive to bacterial growth [50]. In AMs, avirulent mycobacteria induced significantly increased apoptosis compared to virulent strains like Mtb H37Rv [51]. Increased apoptosis in Mtb-infected AMs was recently shown to be NFKB activation-dependent [52]. Serine protease inhibitor 9 (PI-9), an apoptosis-associated gene, was also induced by virulent Mtb, knockdown of which leads to increased expression of the antiapoptotic molecule Bcl-2 and increased production of caspase 3. PI-9 appears to be important in the survival of Mtb within AMs [53]. Interestingly, increased induction of the endoplasmic reticulum (ER) stress pathway, which is directly related to apoptosis, has been noted in human tuberculous granulomas where apoptotic cells accumulated, raising the issue of whether macrophage apoptosis and progressive cell death might be potentially detrimental to the host by causing bacterial dissemination [54]. Thus, despite the evidence supporting the role of apoptosis as a potentially beneficial response in preventing bacterial growth and disease progression, the macrophage-Mtb interaction within the granuloma appears to be more complex, and it remains to be seen whether selective induction of apoptosis in human TB granulomas is protective or detrimental to the host.

\section{Conclusions}

The immunobiology of human alveolar macrophages in protection versus propagation of TB disease continues to be defined. Although, these cells have similarities with monocytederived macrophages, they have unique phenotypic and functional properties which are highly relevant given their intraalveolar location and need to be further delineated in future 
studies. Macrophage plasticity heterogeneity and varying responses between virulent and avirulent mycobacterial strains have generated added complexity to this field. Future immunotherapeutic strategies for control of TB infection will require strain-specific in-depth understanding of the cellular processes that control Mtb uptake, phagolysosome formation, and replication in these cells.

\section{Compliance with Ethics Guidelines}

Conflict of Interest The author reports no conflicts of interest. Because the author is a government employee and this is a government work, the work is in the public domain in the USA. Notwithstanding any other agreements, the NIH reserves the right to provide the work to PubMedCentral for display and use by the public, and PubMedCentral may tag or modify the work consistent with its customary practices. You can establish rights outside of the USA subject to a government use license.

Human and Animal Rights and Informed Consent This article does not contain any studies with human or animal subjects performed by the author.

\section{References}

1. WHO Global tuberculosis report 2014. 2014.

2. Lipscomb MF et al. Human alveolar macrophages: HLA-DRpositive macrophages that are poor stimulators of a primary mixed leukocyte reaction. J Immunol. 1986;136(2):497-504.

3. Li J et al. cDNA microarray analysis reveals fundamental differences in the expression profiles of primary human monocytes, monocyte-derived macrophages, and alveolar macrophages. J Leukoc Biol. 2007;81(1):328-35.

4. Rylance J, et al. Household air pollution causes dose-dependent inflammation and altered phagocytosis in human macrophages. American Journal of Respiratory Cell and Molecular Biology, 2014.

5. Richard N.v.Z.-S, et al, Cigarette smoke and nicotine impair effector responses and mycobacterial stasis in human macrophages, in B25. Tuberculosis: infection and immunity. 2013, American Thoracic Society. p. A2477-A2477.

6. Schwander S, Dheda K. Human lung immunity against Mycobacterium tuberculosis: insights into pathogenesis and protection. Am J Respir Crit Care Med. 2011;183(6):696-707.

7. Merchant RK et al. Bronchoalveolar lavage cellularity. The distribution in normal volunteers. Am Rev Respir Dis. 1992;146(2): 448-53.

8. Ettensohn DB et al. Bronchoalveolar lavage in the normal volunteer subject. I. Technical aspects and intersubject variability. Chest. 1988;94(2):275-80.

9. Herrera MT et al. Compartmentalized bronchoalveolar IFN- $\gamma$ and IL-12 response in human pulmonary tuberculosis. Tuberculosis (Edinburgh, Scotland). 2009;89(1):38-47.

10. Geissmann F et al. Development of monocytes, macrophages, and dendritic cells. Science. 2010;327(5966):656-61.

11. Bitterman PB et al. Alveolar macrophage replication. One mechanism for the expansion of the mononuclear phagocyte population in the chronically inflamed lung. J Clin Invest. 1984;74(2): 460-9.

12. Wahlstrom $\mathbf{J}$ et al. Phenotypic analysis of lymphocytes and monocytes/macrophages in peripheral blood and bronchoalveolar lavage fluid from patients with pulmonary sarcoidosis. Thorax. 1999;54(4):339-46.

13. Pons AR et al. Phenotypic characterisation of alveolar macrophages and peripheral blood monocytes in COPD. Eur Respir J. 2005;25(4):647-52.

14. Viksman MY et al. Phenotypic analysis of alveolar macrophages and monocytes in allergic airway inflammation. I. Evidence for activation of alveolar macrophages, but not peripheral blood monocytes, in subjects with allergic rhinitis and asthma. Am J Respir Crit Care Med. 1997;155(3):858-63.

15. DesJardin LE et al. Mycobacterium tuberculosis-infected human macrophages exhibit enhanced cellular adhesion with increased expression of LFA-1 and ICAM-1 and reduced expression and/or function of complement receptors, FcgammaRII and the mannose receptor. Microbiology. 2002;148(Pt 10):3161-71.

16. Juarez E et al. Differential expression of Toll-like receptors on human alveolar macrophages and autologous peripheral monocytes. Respir Res. 2010;11:2.

17. Mwandumba $\mathrm{HC}$ et al. Alveolar macrophages from HIV-infected patients with pulmonary tuberculosis retain the capacity to respond to stimulation by lipopolysaccharide. Microbes Infect. 2007;9(9): 1053-60.

18. Lay JC et al. In vivo uptake of inhaled particles by airway phagocytes is enhanced in patients with mild asthma compared with normal volunteers. Thorax. 2009;64(4):313-20.

19. Tomlinson GS et al. Adherent human alveolar macrophages exhibit a transient pro-inflammatory profile that confounds responses to innate immune stimulation. PLoS One. 2012;7(6), e40348.

20. Elomaa $\mathrm{O}$ et al. Cloning of a novel bacteria-binding receptor structurally related to scavenger receptors and expressed in a subset of macrophages. Cell. 1995;80(4):603-9.

21. Gordon S, Taylor PR. Monocyte and macrophage heterogeneity. Nat Rev Immunol. 2005;5(12):953-64.

22. Liddiard $\mathrm{K}$ et al. Macrophage heterogeneity and acute inflammation. Eur J Immunol. 2011;41(9):2503-8.

23. Thepen T, Kraal G, Holt PG. The role of alveolar macrophages in regulation of lung inflammation. Ann N Y Acad Sci. 1994;725: 200-6.

24. Fels AO, Cohn ZA. The alveolar macrophage. J Appl Physiol (1985). 1986;60(2):353-69.

25. Crowther JE et al. Pulmonary surfactant protein a inhibits macrophage reactive oxygen intermediate production in response to stimuli by reducing NADPH oxidase activity. J Immunol. 2004;172(11):6866-74.

26. Ferguson JS et al. Surfactant protein D increases fusion of Mycobacterium tuberculosis-containing phagosomes with lysosomes in human macrophages. Infect Immun. 2006;74(12):70059.

27. Ferguson JS et al. Surfactant protein D binds to Mycobacterium tuberculosis bacilli and lipoarabinomannan via carbohydratelectin interactions resulting in reduced phagocytosis of the bacteria by macrophages. J Immunol. 1999;163(1):312-21.

28. Nguyen BY et al. Differences in phagocytosis and killing by alveolar macrophages from humans, rabbits, rats, and hamsters. Infect Immun. 1982;36(2):504-9.

29. Gaynor CD et al. Pulmonary surfactant protein A mediates enhanced phagocytosis of Mycobacterium tuberculosis by a direct interaction with human macrophages. J Immunol. 1995;155(11): 5343-51.

30. Roecklein JA, Swartz RP, Yeager Jr H. Nonopsonic uptake of Mycobacterium avium complex by human monocytes and alveolar macrophages. J Lab Clin Med. 1992;119(6):772-81.

31. Hirsch CS et al. Complement receptor-mediated uptake and tumor necrosis factor-alpha-mediated growth inhibition of Mycobacterium tuberculosis by human alveolar macrophages. J Immunol. 1994;152(2):743-53. 
32. Tailleux L et al. DC-SIGN induction in alveolar macrophages defines privileged target host cells for mycobacteria in patients with tuberculosis. PLoS Med. 2005;2(12), e381.

33. Taha RA et al. IFN-gamma and IL-12 are increased in active compared with inactive tuberculosis. Am J Respir Crit Care Med. 1997;155(3):1135-9.

34. Rich EA et al. Dyscoordinate expression of tumor necrosis factoralpha by human blood monocytes and alveolar macrophages. Am Rev Respir Dis. 1989;139(4):1010-6.

35. Zissel $\mathrm{G}$ et al. In vitro release of interleukin-15 by broncho-alveolar lavage cells and peripheral blood mononuclear cells from patients with different lung diseases. Eur Cytokine Netw. 2000;11(1):105-12.

36. Law $\mathrm{K}$ et al. Increased release of interleukin-1 beta, interleukin-6, and tumor necrosis factor-alpha by bronchoalveolar cells lavaged from involved sites in pulmonary tuberculosis. Am J Respir Crit Care Med. 1996;153(2):799-804.

37. Flynn JL et al. An essential role for interferon gamma in resistance to Mycobacterium tuberculosis infection. J Exp Med. 1993;178(6): 2249-54.

38. Cooke GS et al. Polymorphism within the interferon-gamma/receptor complex is associated with pulmonary tuberculosis. Am J Respir Crit Care Med. 2006;174(3):339-43.

39. Engele $\mathrm{M}$ et al. Induction of TNF in human alveolar macrophages as a potential evasion mechanism of virulent Mycobacterium tuberculosis. J Immunol. 2002;168(3):1328-37.

40. Carranza $\mathrm{C}$ et al. Mycobacterium tuberculosis growth control by lung macrophages and CD8 cells from patient contacts. Am J Respir Crit Care Med. 2006;173(2):238-45.

41. Sadek MI et al. Chemokines induced by infection of mononuclear phagocytes with mycobacteria and present in lung alveoli during active pulmonary tuberculosis. Am J Respir Cell Mol Biol. 1998;19(3):513-21.

42. Silver RF et al. Human alveolar macrophage gene responses to Mycobacterium tuberculosis strains H37Ra and H37Rv. Am J Respir Cell Mol Biol. 2009;40(4):491-504.

43. Mwandumba $\mathrm{HC}$ et al. Mycobacterium tuberculosis resides in nonacidified vacuoles in endocytically competent alveolar macrophages from patients with tuberculosis and HIV infection. $\mathrm{J}$ Immunol. 2004;172(7):4592-8.

44. Bruns $\mathrm{H}$ et al. Abelson tyrosine kinase controls phagosomal acidification required for killing of Mycobacterium tuberculosis in human macrophages. J Immunol. 2012;189(8):4069-78.

45. Borelli $\mathrm{V}$ et al. Ultrastructure of the interaction between mycobacterium tuberculosis- H37Rv-containing phagosomes and the lysosomal compartment in human alveolar macrophages. Exp Mol Pathol. 2002;73(2):128-34.

46. Brooks MN et al. NOD2 controls the nature of the inflammatory response and subsequent fate of Mycobacterium tuberculosis and M. bovis BCG in human macrophages. Cell Microbiol. 2011;13(3): 402-18.

47. Juarez E et al. NOD2 enhances the innate response of alveolar macrophages to Mycobacterium tuberculosis in humans. Eur J Immunol. 2012;42(4):880-9.

48. Juarez E et al. Nucleotide-oligomerizing domain-1 (NOD1) receptor activation induces pro-inflammatory responses and autophagy in human alveolar macrophages. BMC Pulm Med. 2014;14:152.

49. O'Leary S, O'Sullivan MP, Keane J. IL-10 blocks phagosome maturation in mycobacterium tuberculosis-infected human macrophages. Am J Respir Cell Mol Biol. 2011;45(1):172-80.

50. Keane $\mathrm{J}$ et al. Infection by Mycobacterium tuberculosis promotes human alveolar macrophage apoptosis. Infect Immun. 1997;65(1): 298-304.

51. Keane J, Remold HG, Kornfeld H. Virulent mycobacterium tuberculosis strains evade apoptosis of infected alveolar macrophages. J Immunol. 2000;164(4):2016-20.

52. Bai X et al. Inhibition of nuclear factor-kappa B activation decreases survival of mycobacterium tuberculosis in human macrophages. PLoS One. 2013;8(4), e61925.

53. Toossi $Z$ et al. Induction of serine protease inhibitor 9 by Mycobacterium tuberculosis inhibits apoptosis and promotes survival of infected macrophages. J Infect Dis. 2012;205(1):144-51.

54. Seimon TA et al. Induction of ER stress in macrophages of tuberculosis granulomas. PLoS One. 2010;5(9), e12772. 